\title{
Do women and men experience similar barriers to surgical careers worldwide?
}

\author{
I. H. Marks, A. Diaz, M. Keem, K. Daniels, SS. Ladi Seyedian, G. S. Philipo, H. Munir, T. \\ Pomerani, N. Peters, C. Lavy, D. Chang
}

\section{Introduction}

Barriers to female surgeons entering the field are well documented in Australia, the USA and the UK, but how generalizable these problems are to other regions remains unknown.

\section{Methods}

A cross sectional analysis was performed using an electronic, multi-question survey developed by the International Federation Medical Student Association (IFMSA)'s Global Surgery Working Group. The survey was distributed via IFMSA mailing lists, conferences, and social media.

\section{Results}

639 medical students from 75 countries completed the survey. There was a significant negative association between perceived access to postgraduate training and desire to pursue a surgical career in women, but not in men [OR $0.46(0.24-0.87)]$. Academic competitiveness was positively associated in the same population [1.88 (1.04-3.42)]. On subset analysis, high cost of postgraduate training and perceived poor availability of resources were negative barriers to women in LMICs, but not men in LMICs. Resourcing was mostly problematic for Middle Eastern and African women and cost of and access to training in Asian women. The effect of workload on desire to become a surgeon showed no significant differences between men and women, and was only significant in the Americas.

\section{Conclusion}

While there are some similarities between amongst women worldwide, it is important to ensure that cultural assumptions and evidence amassed in high-income country isn't generalized to other regions without evidence of consistency in behaviour or perception. Any strategy to increase the number of students undertaking surgical careers must be considered locally, and a generalized global strategy may prove ineffective. 